\title{
Importance of checking prehospital neurological findings to reveal incidence of spinal cord concussion
}

\author{
Y Yanagawa and H Miyawaki
}

Objective: To clarify the incidence and characteristics of spinal cord concussion in an urban city in Japan.

Methods: The current retrospective study was conducted by reviewing the medical records of patients with spinal cord injuries admitted between January 2007 and December 2010. The subjects were divided into two groups: a spinal cord concussion group and a spinal cord injury group.

Results: There were 36 patients with spinal cord injuries admitted during this period. Among them, there were 8 patients with spinal cord concussions. No significant differences were apparent between the two groups with regard to sex, mechanism of injury, Glasgow Coma Scale, incidence of shock, inhospital Frankel classification, level of spinal cord injury, frequency of vertebral dislocation/fracture, surgery and the survival rate. However, the mean age in the spinal cord concussion group was lower than that in the spinal cord injury group. The systolic blood pressure, the heart rate and the diameter of minimum spinal canal in the concussion group were all higher than those in the spinal cord injury group. Half of the subjects in the spinal cord concussion group demonstrated immediate neurological improvement between prehospital and inhospital findings, however none of the subjects in the spinal cord injury group demonstrated such improvement. This difference was statistically significant.

Conclusion: Spinal cord concussion is not rare in urban Japan. Checking neurological findings at the scene leads to the revelation of the true high incidence of spinal cord concussion among spinal cord injuries.

Spinal Cord (2012) 50, 278-280; doi:10.1038/sc.2011.151; published online 17 January 2012

Keywords: spinal cord concussion; spinal cord injury; prehospital care

\section{INTRODUCTION}

A spinal cord concussion is an injury that causes transient paralysis and/or sensory disturbance, subsiding within 2 or 3 days. ${ }^{1,2}$ Del Bigio et al. ${ }^{1}$ reported that spinal cord concussion represented $6 \%$ of all spinal cord injuries. Because of its rare incidence, there have been few reports concerning spinal cord concussion in English literatures and no reports in Japanese. ${ }^{1-6}$ Previous reports of spinal cord concussion conclude that sports and falls were the leading cause of the injury, and that the most common site affected was the cervical or thoracolumbar junction among young subjects. There was controversy whether pre-existing abnormalities of the spine, such as spinal canal stenosis, can be a risk factor for the occurrence of spinal cord concussion. ${ }^{2-8}$ We therefore investigated the incidence and characteristics of spinal cord concussion at our institute in an urban setting in Japan.

\section{PATIENTS AND METHODS}

This retrospective study protocol was approved by our institutional review board, and examinations were conducted according to the standards of good clinical practice and the Helsinki Declaration. The current study was conducted by reviewing the medical records of patients with spinal cord injuries admitted to our institution between January 2007 and December 2010 at the National Defense Medical College, located in the suburban area of Tokyo, with a neighboring population of approximately 800000 people. The definition of spinal cord concussion was 'transient paralysis and/or sensory disturbance, subsiding within $72 \mathrm{~h}$ '.

The subjects were divided into two groups: a spinal cord concussion group and a spinal cord injury group. The following variables were analyzed between groups: age, sex; mechanism of injury (fall, traffic accident or violence), Glasgow Coma Scale on arrival, systolic blood pressure, heart rate, respiratory rate, incidence of shock, Frankel classification at prehospital and inhospital examination, the level of spinal cord injury, diameter of the spinal canal, existence of spinal canal stenosis, vertebral dislocation/fracture, traumatic lesion at spinal cord detected by magnetic resonance imaging, steroid use, surgical treatment and the survival rate.

Statistical analysis was performed using Student's unpaired $t$-test and the contingency table analyses. Differences with values of $P<0.05$ were considered statistically significant.

\section{RESULTS}

During the investigation period, a total of 746 blunt traumatized patients were admitted to our institution. There were 36 patients with a spinal cord injury. Among these, there were 8 patients with spinal cord concussions.

The results of the comparisons between the groups are shown in Table 1, and details of the spinal cord concussion patients appear in Table 2. No significant differences were apparent between the two groups with regard to sex, mechanism of injury, Glasgow Coma Scale, incidence of shock, Frankel classification, level of spinal cord injury, frequency of vertebral dislocation/fracture, surgical treatment and the survival rate. However, the age and frequency of steroid use in the spinal cord concussion group were lower than those in the spinal cord injury group. The systolic blood pressure, the heart rate and the minimum diameter of the spinal canal were higher than those in the spinal cord injury group. Half of the subjects in the spinal cord concussion group demonstrated immediate neurological improvement 
between prehospital and inhospital findings, however none of subjects in the spinal cord injury group demonstrated such improvement. None of the subjects in the spinal cord concussion group $(n=5)$, who underwent the magnetic resonance imaging study, had traumatic lesions in the spinal cord, however, 22 of the $25(88 \%)$ subjects in

Table 1 Comparison between spinal cord concussion and injury group

\begin{tabular}{|c|c|c|c|}
\hline & $\operatorname{sCC}(n=8)$ & $S C l(n=28)$ & P-value \\
\hline Age (years) & $25.2 \pm 3.0$ & $54.1 \pm 2.9$ & $<0.0001$ \\
\hline Gender (male/female) & $6 / 2(75)$ & 25/3 (89.3) & NS \\
\hline Mechanism of injury & & & NS \\
\hline Fall $(\%)$ & $6(75)$ & $19(67.8)$ & \\
\hline Traffic accident (\%) & $1(12.5)$ & $8(28.5)$ & \\
\hline Violence (\%) & $1(12.5)$ & $1(3.5)$ & \\
\hline Glasgow Coma Scale & $14.0 \pm 0.4$ & $14.4 \pm 0.2$ & NS \\
\hline Systolic blood pressure $(\mathrm{mm} \mathrm{Hg})$ & $132.8 \pm 4.8$ & $110.1 \pm 5.3$ & 0.03 \\
\hline Incidence of shock & 0 & $9(32.1)$ & NS \\
\hline Heart rate (per min) & $93.0 \pm 4.0$ & $77.2 \pm 3.5$ & 0.03 \\
\hline Respiratory rate (per min) & $27.3 \pm 3.2$ & $24.7 \pm 1.6$ & NS \\
\hline Frankel classification & & & NS \\
\hline$A(\%)$ & $1(12.5)$ & $7(25)$ & \\
\hline $\mathrm{B}(\%)$ & 0 & $5(17.8)$ & \\
\hline$C(\%)$ & $5(62.5)$ & $6(21.4)$ & \\
\hline $\mathrm{D}(\%)$ & $2(25)$ & $10(35.7)$ & \\
\hline$E(\%)$ & 0 & $2(7.1)$ & \\
\hline Improvement of neurogenic findings & $4(50)$ & 0 & 0.001 \\
\hline Level of injury & & & NS \\
\hline Cervical & $7(87.5)$ & $23(82.1)$ & \\
\hline Thoracic & $1(12.5)$ & $2(7.1)$ & \\
\hline Lumbar & 0 & $3(10.7)$ & \\
\hline Minimum diameter of spinal canal $(\mathrm{mm})$ & $12.7 \pm 0.8$ & $8.6 \pm 0.5$ & 0.0004 \\
\hline Spinal canal stenosis (\%) & $2(25)$ & $23(82.1)$ & $<0.0001$ \\
\hline Fractures or dislocation (\%) & $1(12.5)$ & $12(42.9)$ & NS \\
\hline Number of SC lesions as detected by MRI & $0(n=5)$ & $22(25)$ & 0.001 \\
\hline Steroid use (\%) & $1(12.5)$ & $16(57.1)$ & 0.04 \\
\hline Surgical treatment (\%) & 0 & $9(32.1)$ & NS \\
\hline Survival rate (\%) & 100 & 96.4 & NS \\
\hline
\end{tabular}

Abbreviations: MRI, magnetic resonance imaging; NS, not significant; SC, spinal cord; SCC, spinal cord concussion; SIC, spinal cord injury. the spinal cord injury group showed spinal cord lesions that were detected by magnetic resonance imaging. These differences were statistically significant.

In the spinal cord concussion group, there were two cases of primary spinal canal stenosis, one cervical disc herniation and one thoraco-lumbar fracture. The cases with disc herniation and burst fracture did not acquire secondary spinal canal stenosis. Four subjects in the spinal cord concussion group had normal radiological findings.

\section{DISCUSSION}

This study demonstrated that spinal cord concussion is not that rare in urban Japan. In addition, patients with spinal cord concussion tended to demonstrate immediate neurological improvement without their vital signs being affected by the vagus nerve.

The incidence of spinal cord concussion among patients with a spinal cord injury in the present study (22\%) was higher than Del Bigio's report $(6 \%) .{ }^{1}$ One of the reasons for these differences may have been differences in prehospital management. For example, some cases with spinal cord concussion were not transferred to a specialized unit. In Japan, there is a JPTEC (Japan prehospital trauma evaluation and care) course that has been centralized under the Japanese Association for Acute Medicine, and the JPTEC council popularized the standard trauma care programs for emergency medical technicians (EMTs) in 2003. ${ }^{9}$ The JPTEC educates EMTs to check patients for paralysis and sensory disturbance, and if the patient was suspected of suffering from a spinal cord injury, then the EMTs were to transport the patients to a level-1 trauma center. Based on the education of the Japanese EMTs, there may have been an increase in the number of spinal cord concussions admitted among the other spinal cord injuries, whereas such patients might have spontaneously resolved when they were admitted to other centers prior to receiving a formal diagnosis of spinal cord concussion. We have treated approximately 9000 patients with clinical emergencies, including trauma, since 1980 . However, we did not diagnose any patients with a spinal cord concussion before 2003. This fact supports our hypothesis. The neurological changes caused by trauma during the acute phase can vary, and are difficult to assess precisely, especially with regard to sensory disturbance, which cannot be evaluated objectively. In addition, most of the patients with spinal cord concussion in this study demonstrated motor weakness, so that recovery of the extremity was simple to evaluate by manual muscle tests, which is in contrast to assessment of sensory disturbance. Scher et $a .^{7}$ reported a study of rugby players with spinal cord

Table 2 Characteristics of patients with spinal cord concussion

\begin{tabular}{|c|c|c|c|c|c|c|c|c|c|c|c|c|c|c|c|}
\hline No. & Age & Sex & Complication & $\begin{array}{c}\text { Diameter of } \\
\mathrm{SCl}(\mathrm{mm})\end{array}$ & Steroid & $\begin{array}{l}\text { Mechanism } \\
\text { of injury }\end{array}$ & $\begin{array}{l}\text { Level of } \\
\text { injury }\end{array}$ & $\begin{array}{l}\text { Frankel } \\
\text { prehospital }\end{array}$ & $\begin{array}{l}\text { Frankel } \\
\text { inhospital }\end{array}$ & $\begin{array}{l}\text { CT } \\
\text { findings }\end{array}$ & $\begin{array}{l}M R I \\
\text { findings }\end{array}$ & GCS & $\begin{array}{c}s B P \\
(m m H g)\end{array}$ & $\begin{array}{c}H R \\
\text { (per min) }\end{array}$ & $\begin{array}{c}R R \\
\text { (per min) }\end{array}$ \\
\hline 1 & 38 & $M$ & $\mathrm{CC}$ & 14 & None & Fall & C & C & C & None & Not performed & 12 & 116 & 90 & 19 \\
\hline 2 & 32 & $\mathrm{~F}$ & $\mathrm{CC}$ & 11.5 & None & Fall & C & $A$ & C & None & Not performed & 15 & 126 & 90 & 30 \\
\hline 3 & 34 & M & None & 10.7 & Use & Fall & $\mathrm{C}$ & $A$ & D & None & None & 15 & 126 & 72 & 30 \\
\hline 4 & 19 & $\mathrm{~F}$ & None & 15.7 & None & Fall & $\mathrm{C} 5$ & $A$ & C & None & Not performed & 15 & 126 & 84 & 18 \\
\hline 5 & 22 & $M$ & None & 11 & None & Traffic & C & $\mathrm{D}$ & $\mathrm{D}$ & None & None & 14 & 128 & 99 & 26 \\
\hline 6 & 25 & $M$ & $\begin{array}{l}\text { Traumatic SAH } \\
\text { Clavicular fx } \\
\text { Scapular fx }\end{array}$ & 17 & None & Fall & Th12 & $A$ & $A$ & Th11-L3 fx & None & 4 & 149 & 98 & 46 \\
\hline 7 & 19 & M & None & 10 & None & Violence & $\mathrm{C}$ & $A$ & C & None & None & 12 & 157 & 104 & 20 \\
\hline 8 & 13 & $M$ & None & 12.3 & None & Fall & $\mathrm{C} 4$ & C & C & None & None & 15 & 135 & 107 & 30 \\
\hline
\end{tabular}

Abbreviations: CC, cerebral concussion; CT, computed tomography; F, female; fx, fracture; GCS, Glasgow coma scale; HR, heart rate; M, male; RR, respiratory rate; SAH, subarachnoid hemorrhage; 
concussion. Their study also indicated a higher frequency of spinal cord concussion among spinal cord injuries (22\%), similar to the present study. In addition, Del Bigio et al. ${ }^{1}$ reported that they experienced four cases with immediate complete recovery of paralysis within $10 \mathrm{~min}$, which usually disappeared in the prehospital setting. Accordingly, checking neurological findings at the scene leads to the revelation of the true high incidence of spinal cord concussion among spinal cord injuries. As some patients with spinal cord concussion can experience a subsidence of symptoms within a few minutes, even EMTs may not see evidence of the neurological abnormality. Thus, the reports provided by those providing first aid or ordinary witnesses may be helpful for evaluating a case with rapid improvement of paralysis due to spinal cord concussion. However, we could not find any official documents describing such patients.

In the present study, there were no patients who demonstrated neurogenic shock in the spinal cord concussion group. In patients with cervical cord injury, bradycardia and hypotension may occur due to suppression of the sympathetic tone following predominance of the vagus. ${ }^{10,11}$ The autonomic nervous system regulates many different target tissues in the somatic and visceral domains of the body in a differentiated manner, indicating that there apparently exist separate sympathetic pathways that are functionally defined by their target cells. ${ }^{12}$ Most of the actions of the autonomic nervous system are involuntary, whereas some activities, such as breathing, can also be controlled by conscious patients. ${ }^{13}$ However, bradycardia or hypotension tended to be reproducible reactions to vagus stimulation, even in patients with spinal cord injury. ${ }^{14,15}$ Neurogenic shock tended to occur more frequently in patients with complete cervical cord injury in comparison with those with incomplete or spinal cord injury without a cervical lesion. ${ }^{11}$ The spinal cord concussion is different from complete spinal cord injury, so patients with a spinal cord concussion do not generally demonstrate neurogenic shock. However, animal studies have shown that severe concussion can cause death via profound autonomic dysfunction, without leaving anatomical evidence of injury, and there was a rare clinical case who may have died due to a vasovagal stimulus after blunt trauma. ${ }^{16,17}$

In patients with a cerebral concussion, electrical, chemical, or ultrastructural changes that likely occur in neurons have yet to be identified. ${ }^{1,17}$ Parkinson speculated that interruption of neural function at the synaptic level may be a feature of concussion. ${ }^{18}$ Comparatively little is known about spinal cord concussion due to the lack of a suitable experimental model. ${ }^{2}$ There were some hypotheses about the mechanism of spinal cord concussion. For example, transient subtle contact with ultrastructural changes between the spinal cord and spinal column may cause spinal cord concussion. Transient contact may be induced by primary or secondary spinal canal stenosis, relaxation of the ligament of the spinal column, ${ }^{8}$ and/or hyperextensive motion that causes the posterior longitudinal ligament and the ligamentum flavum to thicken, the dural diameter to decrease in size and the diameter of the spinal cord to increase in size. ${ }^{4}$ There was also a report suggesting that compression of the spinal cord during hyperextension by infolding of the laminar ligaments resulted in narrowing of the spinal canal. ${ }^{7}$ Groat et al. ${ }^{19}$ emphasized the possibility of a functional block of neural activity. In addition, as transient ischemia causes transient neurological deficits, transient hypoperfusion of the spinal artery may cause a spinal cord concussion. ${ }^{20}$

\section{CONCLUSION}

The frequency of spinal cord concussion was not rare in the present study. Checking neurological findings at the scene leads to the revelation of the true high incidence of spinal cord concussion among spinal cord injuries.

\section{CONFLICT OF INTEREST}

The authors declare no conflict of interest.

1 Del Bigio MR, Johnson GE. Clinical presentation of spinal cord concussion. Spine 1989; 14: 37-40.

2 Zwimpfer TJ, Bernstein M. Spinal cord concussion. J Neurosurg 1990; 72: 894-900.

3 Liao CC, Lui TN, Chen LR, Chuang CC, Huang YC. Spinal cord injury without radiological abnormality in preschool-aged children: correlation of magnetic resonance imaging findings with neurological outcomes. J Neurosurg 2005; 103(1 Suppl): 17-23.

4 Scher AT. Spinal cord concussion in rugby players. Am J Sports Med 1991; 19: 485-488.

5 Rathbone D, Johnson G, Letts M. Spinal cord concussion in pediatric athletes. J Pediatr Orthop 1992; 12: 616-620.

6 Torg JS, Pavlov H, Genuario SE, Sennett B, Wisneski RJ, Robie BH et al. Neurapraxia of the cervical spinal cord with transient quadriplegia. J Bone Joint Surg 1986; 68: $1354-1370$

7 Scher AT. Rugby injuries to the cervical spine and spinal cord: a 10-year review. Clin Sports Med 1998; 17: 195-206.

8 Ferrera PC, Hayes ST, Triner WR. Spinal cord concussion in previously undiagnosed osteogenesis imperfecta. Am J Emerg Med 1995; 13: 424-426.

9 Mashiko K. Trauma systems in Japan: history, present status and future perspectives. J Nippon Med Sch 2005; 72: 194-202.

10 Bilello JF, Davis JW, Cunningham JA, Groom TF, Lemaster D, Sue LP. Cervical spinal cord injury and the need for cardiovascular intervention. Arch Surg 2003; 138: 1127-1129.

11 Ploumis A, Yadlapalli N, Fehlings MG, Kwon BK, Vaccaro AR. A systemic review of the evidence supporting a role for vasopressor support in acute SCI. Spinal Cord 2010; 48: 356-362.

12 Jänig W, Häbler HJ. Neurophysiological analysis of target-related sympathetic pathways-from animal to human: similarities and differences. Acta Physiol Scand 2003; 177: 255-274

13 Mayo KR. Support from neurobiology for spiritual techniques for anxiety: a brief review. J Health Care Chaplain 2009; 16: 53-57.

14 Takahashi M, Matsukawa K, Nakamoto T, Tsuchimochi H, Sakaguchi A, Kawaguchi K et al. Control of heart rate variability by cardiac parasympathetic nerve activity during voluntary static exercise in humans with tetraplegia. J Appl Physiol 2007; 103: 1669-1677.

15 van Lieshout JJ, Wieling W, Karemaker JM, Eckberg DL. The vasovagal response. Clin Sci 1991; 81: 575-586.

16 Davis GG, Glass JM. Case report of sudden death after a blow to the back of the neck. Am J Forensic Med Pathol 2001; 22: 13-18.

17 Taylor AR. The mechanism of injury to the spinal cord in the neck without damage to the vertebral column. J Bone Joint Surg 1951; 33B: 543-547.

18 Parkinson D. The biomechanics of concussion. Clin Neurosurg 1982; 29: 131-145.

19 Groat RA, Rambach Jr WA, Windle WF. Concussion of the spinal cord. An experimental study and a critique of the use of the term. Surg Gynecol Obstet 1945; 81: 63-74.

20 Mulligan JM, Miller T, McGuffie AC, Graham CA. Spinal cord injury without radiographic abnormality in a 4-year-old child: hypoperfusion injury or direct trauma? Eur $\mathrm{J}$ Emerg Med 2007; 14: 216-218. 